\title{
Contents of Volume 50
}

EDitorial

Thomas Fuchs, Fortschritt, Diskontinuität und Einheit der Wissenschaften. Eine Untersuchung zum Problem wissenschaftlicher Polyperspektivität ( Progress, Discontinuity, and Unity of Sciences. The Problem of Scientific Polyperspectivity)

S. Geroulanos, F. Jaggi, J. Wydler, M. Lachat, M. Cakmakci, Thoracopagus symmetricus : Zur Trennung von siamesischen Zwillingen im 10. Jahrhundert n. Chr. durch byzantinische Ärzte (The Separation of Siamese Twins by Byzantine Physicians in the 10th Century A.D.)

Beat Glaus, Rudolf Wolf: Lehrer, Forschungsorganisator und Wissenschaftshistoriker. Zu seinem 100. Todesjahr (Rudolf Wolf: Teacher, Research Organizer, and Historian of Sciences)

Urs B. Leu, Marginalien Konrad Gessners als historische Quelle ( Marginal Notes of Conrad Gessner as a Historical Source)

Bernard Minder, Jung an Freud 1905: Ein Bericht über Sabina Spielrein (Jung to Freud 1905: A Report on Sabina Spielrein)

BEat NäF, Anfänge römischer Medizinkritik und ihre Rezeption in Rom (Early Roman Criticism of Medicine and its Reception in Rome)

Plinio Prioneschi, Skull Trauma in Egyptian and Hippocratic Medicine

Gerhard Rudolph, Erinnerungen an Paul Bert (1833-1886) und die Entwicklung der Höhenphysiologie in der Schweiz (Paul Bert [1833-1886] and the Development of High-Altitude Physiology)

Werner Sackmann, John Tyndall (1820-1893) und seine Beziehungen zu den Alpen und zur Schweiz (John Tyndall [1820-1893], the Alps and Switzerland)

Guy Saudan, Biographie médicale: Fossiles vivants et retour du sujet (Medical Biography: Living Fossils and Return of the Subject)

Michael Stolberg, Das Staunen vor der Schöpfung: «Tota substantia», «calidum innatum», «generatio spontanea» und atomistische Formenlehre bei Daniel Sennert (Marvelling at Creation: Daniel Sennert's Concept of Total Substance, Innate Heat, Spontaneous Generation, and his Theory of the Atoms of Form)

Semir Zeki, The Mystery of Louis Verrey (1854-1916) 


\section{Short Communications}

Thomas Böni, Gründung der Arbeitsgruppe für Klinische Paläopathologie

Christoph Mörgeli, Chirurgischer Eingriff gegen die Übervölkerung: Professor Weinholds Vorhaut-Infibulation (Surgical Action Against Overpopulation: Weinhold's Infibulation of the prepuce)

René Renggli, Harte Drogen - gestern und heute (Hard Drugs - Yesterday and Today)

Obituaries

\section{News AND Activities}

Prof. Dr. med. Huldrych M. Koelbing 70jährig (Boschung/Rüttimann) 130

Veröffentlichungen von Prof. Dr. med. Huldrych M. Koelbing

L'Institut Louis Jeantet d'histoire de la médecine (Université de Genève); Perspectives

et projets - Entretien avec le professeur Bernardino Fantini (Mudry)

Zum Rücktritt von Privatdozent Dr. phil. II Heinz Balmer (Rüttimann) 142

European Research Conferences $\quad 143$

$\begin{array}{ll}\text { Marc-Auguste Pictet Prize } & 144\end{array}$

VI. Internationaler Leibniz-Kongress 144

Schweizerische Gesellschaft für Geschichte der Medizin und der Naturwissenschaften (SGGMN): Vorstand 1993-1995, Jahrestagung 1993

Prof. Dr. Jean Starobinski: member of the Society of Scholars of the Johns Hopkins

University

100 Jahre Pschyrembel

$\begin{array}{ll}\text { Darwin und Darwinismus: kulturhistorische Ausstellung } 1994 \text { in Dresden } & 275 \\ \text { Bicentenaire de la mort d'Antoine-Laurent de Lavoisier } & 275\end{array}$

$\begin{array}{ll}\text { Arbeitskreis Alte Medizin } & 275\end{array}$

34th International Congress on the History of Medicine 1994

Origins and Evolution of Collecting Scientific Instruments 276

XIII Scientific Instrument Symposium 276

Annual Meeting of the Swiss Society of the History of Medicine and Sciences $1993 \quad 276$

Kongress der European Association for the History of Medicine and Health (EAHMH) 277

\section{Essay Reviews}

Erwin H. Ackerknecht, Geschichte der Medizin. 7., überarbeitete und ergänzte Auflage von Axel Hinrich Murken (Koelbing)

Michael R. McVaugh and Nancy G. Siraisi (eds.), Renaissance medical learning : evolution and tradition (Vickers) 


\section{Book Reviews}

Jens Alber, Das Gesundheitswesen der Bundesrepublik Deutschland (Gutzwiller)

Udo Benzenhöfer und Wilhelm Kühlmann (Hrsg.), Heilkunde und Krankheitserfahrung in der frühen Neuzeit (Böni)

Klaus Bergdolt, Der dritte Kommentar Lorenzo Ghibertis. Naturwissenschaften und Medizin in der Kunsttheorie der Frührenaissance (Seiler)

K. van Berkel, M.J. van Lieburg, H. A. M. Snelders, Spiegelbeeld der Wetenschap. Het Genootschaap ter bevordering van Natuur-, Genees- en Heelkunde 1790-1990 (Daems)

Volker Bialas (Hrsg.), Naturgesetzlichkeit und Kosmologie in der Geschichte. Festschrift für Ulrich Grigull (Glaus)

W. F. Bynum and Roy Porter (eds.), Living and dying in London (Hubler)

Ranice W. Crosby, John Cody, Max Brödel, the man who put art into medicine (Stettler)

La defensa del patrimonio histórico-médico español. Actas del III Simposio de la Sociedad Española de Historia de la Medicina (Keller-Schnider)

Antoinette Emch-Dériaz, Tissot, physician of the Enlightenment (Saudan)

Hans-Rudolf Fehlmann, Aus dem Leben und Wirken von Carlos de Gimbernat (17681834) (Koelbing)

Günter B. Fettweis und Günther Hamann (Hrsg.), Über Ignaz von Born und die Societät der Bergbaukunde (Trümpy)

Franz Hermann Franken, Die Krankheiten grosser Komponisten (Weber)

Hermann Fredenhagen, Urs Romer, Beat Rüttimann (Hrsg.), Geschichte der Schweizerischen Gesellschaft für Orthopädie : die zweiten 25 Jahre 1967-1992 (Knessl)

Thomas Fuchs, Die Mechanisierung des Herzens. Harvey und Descartes - der vitale und der mechanische Aspekt des Kreislaufs (Reubi)

Frank Geerk, Paracelsus - Arzt unserer Zeit (Nager)

Wolfgang Götz, Der Briefwechsel von Johann Bartholomäus Trommsdorff (1770-1837) (Schramm)

Michelle Goupil (éd.), Lavoisier et la révolution chimique (Zollinger)

Bernhard Dietrich Haage, Studien zur Heilkunde im «Parzival» Wolframs von Eschenbach (Bergdolt)

Frank Hatje, Leben und Sterben im Zeitalter der Pest (Stettler)

Ernst von Held-Ritt, Prissnitz auf Gräfenberg oder treue Darstellung seines Heilverfahrens mit kaltem Wasser... (Weber)

Geneviève Heller, Charlotte Olivier : la lutte contre la tuberculose dans le canton de Vaud (Koelbing)

Armin Herrmann, Weltreich der Physik: Von Galilei bis Heisenberg (Kiefer)

Jean Imbert (dir.), La protection sociale sous la Révolution Française (Louis-Courvoisier)

Jacques Jouanna, Hippocrate (Zimmermann)

Nahum Kipnis, History of the principle of interference of light (Wiederkehr)

Ernst Kraas und Yoshiki Hiki (Hrsg.), 300 Jahre deutsch-japanische Beziehungen in der Medizin (Gradmann)

Karl Kuhn, Medizinhistorische Institutionen und Publikationen 1991 (Boschung) 
Georgette Legée, Pierre Flourens, 1794-1867 - Physiologiste et historien des sciences (Mayer)

Gunther Lind, Physik im Lehrbuch 1700-1850. Zur Geschichte der Physik und ihrer Didaktik in Deutschland (Kiefer)

Ulrich Lohse, Instrumentenmacher, Dentalfabriken und Dentaldepots: systematisches Verzeichnis von Firmen der Dentalindustrie und des Dentalhandels in Deutschland bis 1938 (Sigron)

Médecine, lettres \& politiques. Equinoxe, revue romande de sciences humaines no. 8, automne 1992 (Müller)

Rudolf Mumenthaler, "Keiner lebt in Armuth». Schweizer Ärzte im Zarenreich (Henning)

Red-hair medicine: Dutch-Japanese medical relations. Ed. by H. Beukers, A. M. Luyendijk-Elshout, M.E. van Opstall and F. Vos (Huizink)

Olivier Rieppel, Unterwegs zum Anfang. Geschichte und Konsequenzen der Evolutionstheorie (Schmutz)

Catrien Santing, Geneeskunde en humanisme: en intellectuele biografie van Theodoricus Ulsenius (c. 1460-1508) (Luyendijk-Elshout)

Siegfried Scheidegger, Tertiäre Syphilis im 16. Jahrhundert (Etter)

Hans-Werner Schütt, Eilhard Mitscherlich, Baumeister am Fundament der Chemie (Kauertz)

La Société Philomathique de Paris et deux siècles de la science en France (Speziali)

Lazzaro Spallanzani, Edizione nazionale delle opere, parte prima Carteggi, a cura di Pericle di Pietro (Boschung)

Th. J. A. Terlouw, De opkomst van het heilgymnastisch beroep in Nederland in de 19de eeuw (Huizink)

Álvaro Martínez Vidal, Neurociencias y revolución cientifica en España (Engeler)

André Weil, Zahlentheorie. Ein Gang durch die Geschichte, von Hammurapi bis Legendre (Burckhardt)

Claude E. Welch, A twentieth-century surgeon. My life in the Massachusetts General Hospital (Naef)

Book Notes 